\title{
AN EXTENSION THEOREM FOR INTEGRAL REPRESENTATIONS
}

\author{
WOLFGANG KNAPP and PETER SCHMID
}

(Received 5 January 1996; revised 11 September 1996)

Communicated by L. Kovács

\begin{abstract}
By a fundamental theorem of Brauer every irreducible character of a finite group $G$ can be written in the field $\mathbb{Q}\left(\varepsilon_{m}\right)$ of $m$ th roots of unity where $m$ is the exponent of $G$. Is it always possible to find a matrix representation over its ring $\mathbb{Z}\left[\varepsilon_{m}\right]$ of integers? In the present paper it is shown that this holds true provided it is valid for the quasisimple groups. The reduction to such groups relies on a useful extension theorem for integral representations. Iwasawa theory on class groups of cyclotomic fields gives evidence that the answer is at least affirmative when the exponent is replaced by the order, and provides for a general qualitative result.
\end{abstract}

1991 Mathematics subject classification (Amer. Math. Soc.): primary 20C10; secondary 11R23, 11R33, $20 \mathrm{C} 34$.

Keywords and phrases: Integral representations, Clifford theory, class groups.

\section{Introduction}

Let $G$ be a finite group with exponent $m=\exp (G)$, and let $\varepsilon_{m}=e^{2 \pi i / m}$. Cliff, Ritter and Weiss [1] proved that if $G$ is solvable every irreducible character of $G$ can be realized over $\mathbb{Z}\left[\varepsilon_{m}\right]$. Their proof is based on Dade's extension theorem [5] for groups with normal extra special $p$-subgroups (which is rather deep for $p=2$ ). In this note we propose an alternate approach and investigate the realization problem for arbitrary finite groups.

The following observation on integral representations will be crucial; it eventually allows the reduction either to extra special groups ( $G$ solvable) or to quasisimple groups. Let firstly $R$ be any Dedekind domain.

(C) 1997 Australian Mathematical Society 0263-6115/97\$A2.00+0.00 
THEOREM 1. Suppose $G$ is a normal subgroup of some (finite) group $H$ and $\rho: G \rightarrow \mathrm{GL}_{d}(R)$ is a representation with absolutely irreducible character $\chi$. Assume $\rho$ is $H$-stable and $\chi$ can be extended to an $R$-valued character $\chi^{\prime}$ of $H$. Then there is a unique representation $\rho^{\prime}: H \rightarrow \mathrm{GL}_{d}(R)$ extending $\rho$ and affording $\chi^{\prime}$.

As usual, an $R G$-module $U$ is called $H$-stable provided all conjugates satisfy $U^{h} \cong U(h \in H)$. So $\rho$ is $H$-stable if there are $T_{h} \in \mathrm{GL}_{d}(R)$ such that $\rho\left(h^{-1} g h\right)=$ $T_{h}{ }^{-1} \rho(g) T_{h}$ for all $g \in G$. This is a necessary condition for $\rho$ to be extendable to $H$.

In order to apply Theorem 1 to our problem, arguing by induction, one has to find stable integral representations affording some given invariant irreducible (complex) character $\chi \in \operatorname{Irr}(G)$. In general this might fail, even for principal domains whose quotient field is a splitting field for $\chi$ (for example, see [17, 19]). Fortunately this difficulty disappears in the presence of suitable roots of unity. Let $n=(m,|G| / d)$ be the greatest common divisor of $m=\exp (G)$ and $|G| / d$ where $d=\chi(1)$ is the degree of $\chi$. Let $R=\mathbb{Z}\left[\varepsilon_{n}\right]$ be the ring of integers in $K=\mathbb{Q}\left(\varepsilon_{n}\right)$.

THEOREM 2. Whenever $G$ is a normal subgroup of some group $H$ and $\chi \in \operatorname{Irr}(G)$ is $H$-invariant, there is a representation group for $\chi$ over $K$ with regard to $H$. In particular, there exists an $H$-stable $R G$-lattice $U$ such that $K \otimes_{R} U$ affords $\chi$.

It is known that $K$ is a splitting field for $\chi[18, T h m .8]$. This interesting addendum to Brauer's celebrated theorem is based on the fact, due to Feit, that the conductor, $f(\chi)$, of the field $\mathbb{Q}(\chi)$ of character values is a divisor of $n=n(\chi)$, and on certain properties of the Schur index. The existence of a representation group for $\chi$ over $K$ enables us to apply the Fong reductions (Clifford theory). So combining Theorems 1 and 2 we may infer that it suffices to solve the problem in case $G$ is a quasisimple group (cf. the proposition in Section 5). In the case that $G$ is solvable, the reduction (to extra special groups) even works for slightly smaller rings of algebraic integers yielding an improvement of the result of Cliff, Ritter and Weiss (see Theorem 3 ).

Note that the module $U$ in Theorem 2 need not be $R$-free. Since the representation theory of the quasisimple groups (in contrast to the extra special groups) is not yet in a shape to benefit from it directly, one is urged to proceed in a different way. We investigate the Steinitz class $[U]$ of $U$ more closely. This is an element of the class group $\mathrm{Cl}_{K}$ of $K$. The idea is to search for (cyclotomic) extension fields $L$ such that $[U]$ becomes trivial or, more generally, a $d$ th power in $\mathrm{Cl}_{L}$. Then we indeed will have realizability of $\chi$ over the ring of integers of $L$.

In fact, the explicit version of Iwasawa theory on class groups of cyclotomic fields obtained in [11] yields the following qualitative result.

THEOREM 3' Given any group $H$ operating on $G$ and leaving $\chi$ invariant there is an integer $n^{\prime} \geq n$, which is divisible only by primes dividing $|G|$ and is independent 
of $H$, such that there is an $H$-stable representation over $\mathbb{Z}\left[\varepsilon_{n^{\prime}}\right]$ affording $\chi$.

Of course, in Theorem $3^{\prime}$ we would like to specify $n^{\prime}=n$ or $n^{\prime}=m$. There is some evidence that one can take at least $n^{\prime}=|G|$. This is substantiated by appropriate behaviour of class groups (and the very choice of $n=n(\chi)$ dividing $|G| / d$ ). We checked this for various (quasisimple) groups $G$, including all sporadic simple groups and their covering groups (using work of Feit [6, 7], the ATLAS [2], and GAP [9]). In order to confirm the choice $n^{\prime}=|G|$ in general we would require more precise information on class groups in cyclotomic Iwasawa towers at low levels.

\section{Proof of Theorem 1}

Let $K$ be the quotient field of $R$. Uniqueness of $\rho^{\prime}$ (affording $\chi^{\prime}$ ) is straightforward; for if $T \in \mathrm{GL}_{d}(K)$ satisfies $T^{-1} \rho(g) T=\rho(g)$ for all $g \in G$, then $T$ is a scalar matrix by Schur's lemma. Note that $\chi$ is assumed to be absolutely irreducible.

In order to prove existence of $\rho^{\prime}$, let $V$ be a $K H$-module affording $\chi^{\prime}$. By a basic property of Schur indices, such a module $V$ exists since $\chi^{\prime}$ is $K$-valued and $\chi=\left(\chi^{\prime}\right)_{G}$ is realizable over $K$ (use Frobenius reciprocity). By hypothesis there is an $H$-stable $R$-free $R G$-lattice $U \subseteq V$ affording $\chi$. Fix an element $h \in H$ and consider the $K$-automorphism $\tilde{h}: v \mapsto v h$ of the (right) $K H$-module $V(v \in V)$.

By assumption $U$ and $U h=U \widetilde{h}$ are isomorphic $R G$-lattices. Since $V_{G}$ is absolutely irreducible and $K \otimes_{R} U=V$, this forces $U h=U c$ for some $c \in K^{*}$ by Schur's lemma. Moreover, $\Lambda=\left\{f \in \operatorname{End}_{K}(V): U f \subseteq U\right\}$ is a maximal $R$-order, isomorphic to $\mathrm{M}_{d}(R)$, in $\operatorname{End}_{K}(V)[3,(26.25)]$. We infer that $\widetilde{h}=c f=f c$ for some $f \in \Lambda$. We must have $U f=U$ for this $f$. Let $t$ denote the order of $h$ in $H$. Then $\widetilde{h}^{t}=f^{t} c^{t}$ is the identity on $V$ and consequently $U=U \tilde{h}^{t}=U f^{t} c^{t}=U c^{t}$. It follows that $c^{t}$ and $c^{-t}$ are integral over $R$. Since $R$ is integrally closed (in $K$ ), we see that $c^{t}$ and $c^{-t}$ belong to $R$. Hence $c$ and $c^{-1}$ belong to $R$. Thus $c$ is a unit in $R\left(c \in R^{*}\right)$ and so $U h=U c=U$. We have established that $U$ carries the structure of an $R H$-lattice (within $V$ ), which proves the theorem.

REMARK. By basic Clifford theory one can associate with $\rho$ (being $H$-stable) a cohomology class $\omega \in \mathrm{H}^{2}\left(H / G, R^{*}\right)$ which vanishes if and only if $\rho$ can be extended to a representation of $H$ over $R$. Since by assumption $\chi$, the character afforded by $\rho$, can be extended to a $K$-valued character of $H$, its Clifford obstruction $\omega_{K H}(\chi)$ is trivial (cf. [4, Prop. 1.5] or [20, Lemma 0]). This obstruction is the natural image of $\omega$ in $\mathrm{H}^{2}\left(H / G, K^{*}\right)$. The long exact cohomology sequence yields the exact sequence

$$
\operatorname{Hom}\left(H / G, K^{*} / R^{*}\right) \rightarrow \mathrm{H}^{2}\left(H / G, R^{*}\right) \rightarrow \mathrm{H}^{2}\left(H / G, K^{*}\right) .
$$

In order to show that $\omega$ vanishes it suffices to check that the Hom-group on the left is trivial. Now observe that $K^{*} / R^{*}$ is torsion-free since $R$ is integrally closed. 


\section{Proof of Theorem 2}

Recall that $n=(m,|G| / d)$ is the greatest common divisor of $m=\exp (G)$ and $|G| / d$ where $d=\chi(1)$ is the degree of the $H$-stable character $\chi \in \operatorname{Irr}(G)$. Recall also that $K=\mathbb{Q}\left(\varepsilon_{n}\right)$ is a splitting field for $\chi\left[18\right.$, Thm. 8]. Let $\omega=\omega_{K H}(\chi)$ be the Clifford obstruction to $\chi$ with regard to $H$ (and $K$ ). We have to show that there is a cocycle of finite order representing this cohomology class (see [20] for a detailed discussion of the concept of representation groups).

There is a finite field extension $L \supseteq K$ such that the corresponding statement holds for the image $\omega_{L H}(\chi)$ of $\omega$ in $\mathrm{H}^{2}\left(H / G, L^{*}\right)$. (One may take for instance $L=\mathbb{Q}\left(\varepsilon_{m}\right)[20]$.) Choose $L$ such that the degree $[L: K]$ is as small as possible. Let $C \mapsto \widetilde{H} \rightarrow H / G$ be a representation group for $\chi$ over $L$ for some group $C$ of roots of unity in $L$ (so the cohomology class of the central extension maps onto $\omega_{L H}(\chi)$ ). Let $\widehat{H}=\widetilde{H} \wedge H$ be the fibre-product of $\widetilde{H}$ and $H$ amalgamating $H / G$. Regarding $\widehat{H}$ as a subgroup of the direct product $\widetilde{H} \times H$, we have a group extension

$$
C \times G \mapsto \widehat{H}=\widetilde{H} \wedge H \rightarrow H / G,
$$

and we may view $\chi$ as a character of $\operatorname{Ker}(\widehat{H} \rightarrow \widetilde{H})=1 \times G$. By the very construction there is an $L$-character (that is, realizable over $L$ ) $\widehat{\chi}$ of $\widehat{H}$ extending $\chi$ viewed this way.

We are going to show that $L=K$. It is enough to find $L, \widetilde{H}$ such that $C \subseteq K^{*}$ and $K(\widehat{x})=K$. For let then $\lambda$ be the unique linear constituent of $\widehat{\chi}_{C \times 1}$. This may be viewed as a $K$-character of $C$. Let $\omega_{\lambda}=\omega_{K \widetilde{H}}(\lambda)$. Then $\widehat{\chi}_{C \times G}=\lambda \otimes \chi$ is an irreducible $K$-character of $C \times G$ with trivial Clifford obstruction $\omega_{\lambda} \cdot \omega$ in $\mathrm{H}^{2}\left(H / G, K^{*}\right.$ ) (cf. [4, Prop. 3.6]). Therefore $\omega=\omega_{\lambda}^{-1}\left(=\omega_{\lambda^{-1}}\right)$ can be represented by a cocycle of finite order [20, Lemma 4].

By restriction to Sylow subgroups we may assume that $P=H / G$ is a $p$-group for some prime $p$. Then also $C$ may be taken of $p$-power order. By [20, Prop. 2] we may assume that $p$ is a divisor of $|G|$ and that $K(\supseteq \mathbb{Q}(\chi))$ contains the $p$ th roots of unity (for otherwise $\omega$ vanishes).

\section{CASE 1. $p$ is odd}

Here we may choose $L$ as a subfield of the field obtained from $K$ by adjoining the $q$ th roots of unity for all primes $q \equiv 1(\bmod p)$ which divide $|G|($ cf. [4, Thm. 6.5] and [20, Prop. 2]). Then $C \subseteq K^{*}$. On the other hand, $K=\mathbb{Q}\left(\varepsilon_{n}\right)$ contains the $q$ th roots of unity for all (odd) primes $q \neq p$ dividing $|G|$ unless $\chi$ is of $q$-defect 0 (and hence $q$-rational). If $\chi$ is of $q$-defect 0 for such a prime $q \neq p$, so is $\widehat{\chi}$, because both $C$ and $P$ are $p$-groups. We conclude that $K(\widehat{\chi})=K$ and $L=K$. 
CASE 2. $p=2$

If $m=\exp (G)$ is not divisible by 4 or $K$ contains the 4 th roots of unity, the result follows exactly as before. Otherwise $\chi$ is of 2-defect 0 or 1 , by definition of $K$. It is shown in [21] that there is, then, a 2-rational representation group for $\chi$, that is, $L$ may be chosen as a subfield of $\mathbb{Q}\left(\varepsilon_{u}\right)$ for some odd integer $u$ dividing $|G|$ (so that $|C|=2$ ). From [18, Thm. 3] it follows (by induction on $|P|$ ) that $[K(\widehat{\chi}): K]$ is a 2-power. But by construction $K$ contains the $q$ th roots of unity for all odd prime divisors $q$ of $|\widehat{H}|$ unless $\widehat{\chi}$ is of $q$-defect 0 . Consequently $K(\widehat{\chi})=K$ and hence $L=K$ again.

As for the additional statement in Theorem 2, observe that $\widehat{\chi}$ can be written in $K$. Thus there is an $R \widehat{H}$-lattice $\widehat{U}$ such that $K \otimes_{R} \widehat{U}$ affords $\widehat{\chi}$ [3, (23.7)]. This $\widehat{U}$ admits a projective representation of the quotient group $H=\widehat{H} /(C \times 1)$ whose factor set depends only on cosets $(\bmod G)$ and has its values in $C$. Restricting this projective representation to $G$ yields an $H$-stable $R G$-lattice $U$ as desired.

REMARK. There is an $H$-stable $R G$-lattice $U$ such that $K \otimes_{R} U$ affords $\chi$ just because $\chi$ can be written in $K$ and $\omega=\omega_{K H}(\chi)$ can be represented by a cocycle having its values in $R^{*}=\mathbb{Z}\left[\varepsilon_{n}\right]^{*}$ (see [17, Lemma 1]; a similar statement, attributed to A. Fröhlich, can be found in [19]). We may then view $\omega$ as an element of $\mathrm{H}^{2}\left(H / G, R^{*}\right)$.

\section{Solvable groups}

In this section we assume that $G$ is solvable. Here we have a quite strong result improving that of Cliff, Ritter and Weiss [1]. We define the Solomon number $s=s(\chi)$ of $\chi \in \operatorname{Irr}(G)$, having degree $d=\chi(1)$, by

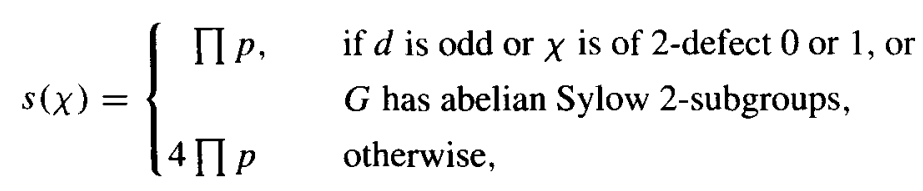

where the product is taken over all odd primes $p$ dividing $|G| / d$ for which $p-1$ divides $d$ or $\chi$ is not $p$-rational. We call the ring $\mathrm{S}(\chi)$ of algebraic integers in the field $K=\mathbb{Q}(\chi)\left(\varepsilon_{s}\right)$ the Solomon ring of $\chi$; this is motivated by work of Solomon [23] (cf. [19] for a similar notion).

It is conjectured that the quotient field $K$ of $\mathrm{S}(\chi)$ is always a splitting field for $\chi$. However, we can establish this only when $G$ is solvable. Observe that $K \subseteq \mathbb{Q}\left(\varepsilon_{n}\right)$ (with $n=n(\chi)$ as above) and that $K \subseteq \mathbb{Q}\left(\varepsilon_{f}\right)$ if $d$ is odd, where $f=f(\chi)$ is the conductor of $\mathbb{Q}(\chi)$. 
THEOREM 3. Suppose $H$ is an operator group of the solvable group $G$ leaving $\chi \in \operatorname{Irr}(G)$ invariant. Then there is an $H$-stable representation $\rho: G \rightarrow \mathrm{GL}_{d}(S)$ affording $\chi$ where $S=\mathrm{S}(\chi)$ is the Solomon ring of $\chi$.

PROOF. Without loss we may assume that $G$ is a normal subgroup of the (finite) group $H$. Put $s=s(\chi)$ and let $K$ be the quotient field of $S$. We argue by induction assuming that $G$ is a (solvable) counterexample of minimal order; the structure of the group $H$ will play no role.

By the choice of $G$ we know that $\chi$ is faithful; note that $\operatorname{Ker}(\chi)$ is normal in $H$ as $\chi$ is $H$-stable. Also, $G$ is not cyclic. We proceed in several steps.

STEP 1: For every $H$-invariant subgroup $N$ of $G$ there is a faithful $\zeta \in \operatorname{Irr}(N)$ such that the restriction $\chi_{N}=e \operatorname{Tr}_{K(\zeta) / K)}(\zeta)$, where $e[K(\zeta): K]$ is a divisor of $|G / N|$.

In this step we use the first Fong reduction (Clifford's theorem). Let $\zeta \in \operatorname{Irr}(N)$ be a constituent of $\chi_{N}$, and let $T, T_{0}$ be the inertia groups of $\zeta$ in $G$ and $H$, respectively. Then $T=G \cap T_{0}$ and $H=G T_{0}$ (by a Frattini argument). Define

$$
\widetilde{T}_{0}=\left\{h \in H: \zeta^{h} \text { and } \zeta \text { are Galois conjugate over } K\right\},
$$

and let $\widetilde{T}=G \cap \widetilde{T}_{0}$. By a standard argument $T, T_{0}$ are normal subgroups of $\widetilde{T}, \widetilde{T}_{0}$, respectively, and

$$
\widetilde{T}_{0} / T_{0} \simeq \operatorname{Gal}(K(\zeta) / K) \simeq \widetilde{T} / T
$$

Let $\theta \in \operatorname{Irr}(T)$ be the homogeneous (or Wedderburn) component of $\chi$ with respect to $\zeta$, and let $\widetilde{\theta}=\theta^{\widetilde{T}}$ be the induced character of $\widetilde{T}$. Then $K(\widetilde{\theta})=K$ and $\chi=\widetilde{\theta}^{G}$. Moreover, $\theta$ is $T_{0}$-stable as $\chi$ is $H$-stable, whence $\widetilde{\theta}$ is $\widetilde{T}_{0}$-stable (as $\widetilde{T}_{0}=\widetilde{T} \cdot T_{0}$ ). We next check that $s(\widetilde{\theta})$ is a divisor of $s=s(\chi)$. Let $\widetilde{d}=\widetilde{\theta}(1)$ so that $d=\tilde{d} \cdot|G: \widetilde{T}|$. If $4 \mid s(\widetilde{\theta})$ then $4 \mid s$. Assume there is an odd prime $p$ dividing $s(\widetilde{\theta})$ but not $s$. Then $p$ divides $|G| / d=|\widetilde{T}| / \widetilde{d}$. Thus $\chi$ is $p$-rational and $p-1$ does not divide $d$. It follows that $K \subseteq \mathbb{Q}\left(\varepsilon_{r}\right)$ for some integer $r$ prime to $p$, and from $K=K(\widetilde{\theta})$ we infer that $\widetilde{\theta}$ is $p$-rational as well. But then $p-1$ must divide $\widetilde{d}$ and hence $d$, a contradiction.

Assume $\widetilde{T} \neq G$. Then, by induction, there is a $\widetilde{T}_{0}$-stable representation $\widetilde{\rho}$ of $\widetilde{T}$ over $S$ affording $\tilde{\theta}$. But then $\rho=\widetilde{\rho}^{G}$ is an $H$-stable representation of $G$ as required. Thus $\widetilde{T}=G$ and $\chi_{N}$ has the stated properties; $\zeta$ is faithful as $\chi$ is faithful and Galois conjugate characters have the same kernel. Finally, it is known that the ramification index $e=\left(\chi_{N}, \zeta\right)$ is a divisor of $|T / N|$.

As a consequence of Step 1, every abelian $H$-invariant subgroup of $G$ is cyclic. In particular, $G$ is not abelian. We now fix a minimal non-abelian $H$-invariant subgroup $E$ of $G$. Let $E / Z$ be a chief factor of $H$. Since $G$ is solvable, $E / Z$ is an elementary abelian $p$-group for some prime $p$ and $Z$ is cyclic (by the choice of $E$ ). 
STEP 2: Either $E$ is an extra special p-group of exponent $p>2$ and $K=K\left(\varepsilon_{p}\right)$ or $p=2$, $\exp (E)=4$ and $K=K\left(\varepsilon_{4}\right)$.

Assume $E$ is not a $p$-group. Let $q \neq p$ be a prime dividing $|Z|$ and $N$ the subgroup of $Z$ of order $q$. Then $N$ is not central in $E$, by minimality of $E$ (and the Schur-Zassenhaus theorem). Let $\zeta \in \operatorname{Irr}(N)$ be a constituent of $\chi_{N}$ and $T=\mathrm{C}_{G}(N)$ its inertia group, which is $H$-invariant. Then $T \cap E=Z$ and, in view of Step 1, $G / T \simeq \operatorname{Gal}\left(K\left(\varepsilon_{q}\right) / K\right)$. Thus $K\left(\varepsilon_{q}\right) \neq K$ and, by definition of the Solomon field, $q$ does not divide $s=s(\chi)$. Clearly $q$ is odd. Since $N$ is a normal subgroup of $G$ of order $q, \chi$ is not of $q$-defect 0 and so $q$ divides $|G| / d$. As $q$ does not divide $s$, by definition of the Solomon number, $\chi$ is $q$-rational. It follows that $|G / T|=\left[K\left(\varepsilon_{q}\right): K\right]=q-1$ is a divisor of $d$, because $\chi$ is induced from some character of $T$. However, this forces $q \mid s$.

This contradiction shows that $E$ is indeed a $p$-group. It follows from Step 1 (with $N=E$ ) that $\chi$ is not of $p$-defect 0 nor 1 . (Alternately use that $\mathrm{O}_{p}(G)$ is contained in the defect groups of every $p$-block of $G$.) Considering the subgroup of order $p$ in $Z$, we obtain that $p \mid s$ and hence $\varepsilon_{p} \in K$. In case $p=2$, even $4 \mid s$, and therefore $\varepsilon_{4} \in K$. This implies that every $H$-invariant subgroup of $E$ of order $p$ (respectively 4 in case $p=2$ ) is central in $G$ (even in $H$ as $\chi$ is $H$-stable).

Since every proper $H$-invariant subgroup of $E$ is cyclic, P. Hall's characterization of the $p$-groups of symplectic type applies [15, III.13.10]. This yields that $E=\Omega_{1}(E)$ is an extra special $p$-group of exponent $p>2$ or $p=2$ and $E$ is either extra special or the central product of an extra special 2-group with a cyclic group of order 4 . (In case $H$ is solvable the latter could be excluded.)

STEP 3: $G=E$ (with $E$ as in Step 2).

Assume the contrary, and let $\zeta \in \operatorname{Irr}(E)$ be a constituent of $\chi_{E}$. Let $m=\exp (E)$ and $K_{0}=\mathbb{Q}\left(\varepsilon_{m}\right) . K_{0}$ is a splitting field for $\zeta$ (Brauer), and we know from Step 2 that $K_{0} \subseteq K$. In view of Step $1, \zeta$ is $G$-stable; it is even $H$-stable as $\chi$ is $H$-invariant.

Now we apply the second Fong reduction and consider the distinguished representation group $H(\zeta)$ of $\zeta$ with regard to $H$ (in the sense of [20]). This is the unique, up to equivalence, central extension $C \longmapsto H(\zeta) \rightarrow H / E$ where $C=\left\langle\varepsilon_{m}\right\rangle$ and where the cohomology class maps onto $\omega_{K_{0} H}(\zeta)$. As above we denote by $\widehat{H}=H(\zeta) \wedge H$ the fibre-product amalgamating $H / E$. By construction there is a $K_{0}$-character $\widehat{\zeta}$ of $\widehat{H}$ which extends $\zeta$ viewed as a character of $1 \times E$. Fix this character.

$\widehat{H}$ contains $\widehat{G}=G(\zeta) \wedge G$ (constructed similarly) as a normal subgroup. By basic Clifford theory there is a unique character $\chi^{\prime}$ of $\widehat{G}$ having $1 \times E$ in its kernel such that $\chi=\widehat{\zeta}_{\widehat{G}} \cdot \chi^{\prime}$ as a character of $\widehat{G}$ (having $C \times 1$ in its kernel). One knows that $\chi$ and its Clifford (or Fong) correspondent $\chi^{\prime} \in \operatorname{Irr}(G(\zeta))$ have the same Schur index over $K_{0}$ and that $K_{0}\left(\chi^{\prime}\right)=K_{0}(\chi)$. (There is a Morita equivalence between 
corresponding irreducible modules over $K_{0}$ yielding isomorphic endomorphism rings.) In particular, $K_{0}\left(\chi^{\prime}\right) \subseteq K=K(\chi)$. Since $\widehat{\zeta}(1)=\zeta(1)$ is a $p$-power (dividing $|E|$ ) and $\chi^{\prime}(1)=d / \zeta(1)$, we readily see that $\mathrm{S}\left(\chi^{\prime}\right) \subseteq S=\mathrm{S}(\chi)$. As $\chi$ and $\widehat{\zeta} \widehat{\mathrm{\sigma}}$ are $\widehat{H}$-stable so is $\chi^{\prime}$ (by uniqueness).

Observe that $|G(\zeta)|<|G|$. There is thus an $S$-free $S \widehat{G}$-lattice $U^{\prime}$ affording $\chi^{\prime}$ which is stable under $\widehat{H}$. A similar statement holds for $\zeta$ (as $|E|<|G|$ ); application of Theorem 1 then yields that there is an $S$-free $S \widehat{H}$-module $\widehat{U}$ affording $\widehat{\zeta}$. But now $U=\widehat{U}_{\widehat{G}} \otimes_{S} U^{\prime}$ is an $S$-free $S G$-lattice which affords $\chi$ and is $H$-stable.

\section{STEP 4: Completion of the proof.}

In the notation used above (with $G=E, \chi=\zeta, K=K_{0}, S=\mathbb{Z}\left[\varepsilon_{m}\right]$ ), there is a $K \widehat{H}$-module $\hat{V}$ affording $\widehat{\chi}=\widehat{\zeta}$. Choose any full $S \widehat{H}$-lattice $\widehat{U} \subseteq \widehat{V}$ and let $U$ be its restriction to $1 \times G$. Regarding $U$ as an $S G$-lattice it is $H$-stable, and $K \otimes_{S} U$ affords $\chi$. This $U$ need not be $S$-free. However, using that $G=E$ is an M-group, there is also an $S$-free $S G$-lattice $W$ affording $\chi$. Without loss we may assume that $U \subseteq W$ (in the $K$-vector space $\widehat{V}$ ).

Now we proceed as in $[1$, Lemma 1.5$]$, studying the order ideal of $W / U$. Using that $W / I W$ is an irreducible $(S / I) G$-module for any maximal ideal $I$ of $S$ not dividing $p$ and that $\pi=S\left(1-\varepsilon_{m}\right)$ is the unique prime ideal in $S$ over $p$, we find an ideal $J$ of $S$ such that $U^{\prime}=J^{-1} U$ is still contained in $W$ but every $S$-composition factor of $W / U^{\prime}$ is isomorphic to $S / \pi\left(\cong \mathbb{F}_{p}\right)$. By the Invariant Factor Theorem for lattices over Dedekind rings, $U^{\prime}$ and $W$ have the same Steinitz class. Hence $U^{\prime}$ is $S$-free likewise. Clearly $U^{\prime}$, which is in the genus of $U$, is $H$-stable and affords $\chi$, as desired.

\section{Genus and Steinitz classes}

We would like to prove Theorem 3 for arbitrary $G$, at least when the Solomon ring $\mathrm{S}(\chi)$ of the character $\chi$ is replaced by $\mathbb{Z}\left[\varepsilon_{|G|}\right]$. Heading for this goal, we take in the sequel again $K=\mathbb{Q}\left(\varepsilon_{n}\right)$ as base field, where as usual $n=(m,|G| / d)$ is the greatest common divisor of $m=\exp (G)$ and $|G| / d$ (with $d=\chi(1)$ ). Let $R=\mathbb{Z}\left[\varepsilon_{n}\right]$ be the ring of integers of $K$.

PROPOSITION. Assume there is an $H$-stable representation $\rho: G \rightarrow \mathrm{GL}_{d}(R)$ affording $\chi$ whenever $G$ is quasisimple and $H$ is an operator group of $G$ stabilizing $\chi$. Then the same holds true in general.

PROOF. Let $G$ be an (arbitrary) normal subgroup of some finite group $H$ stabilizing $\chi$. We argue by induction. By Theorem 3 we may assume that $G$ is not solvable. For any ( $H$-invariant) normal subgroup $N$ of $G$ and any irreducible constituent $\zeta$ of 
$\chi_{N}$, by Clifford's theorem (and known properties of the ramification index) $|N| / \zeta(1)$ is a divisor of $|G| / \chi(1)$. Clearly $\exp (N)$ divides $\exp (G)$; a similar statement holds for representation groups with regard to $G$ [20]. Hence, by virtue of Theorem 2, both Fong reductions work (see Steps 1 and 3 of the proof of Theorem 3 ).

Let now $E$ be a minimal non-abelian $H$-invariant subgroup of $G$ and let $\zeta \in \operatorname{Irr}(E)$ be a constituent of $\chi_{E}$. Combining the Fong reductions and Theorem 1, we are at once reduced to the situation where $\zeta=\chi_{E}$ is faithful and the center $Z=\mathrm{Z}(E)$ is cyclic and central in $H$. If $E$ were solvable or $|E|<|G|$, the result would follow from Theorem 3 or by induction, on the basis of Theorem 1. (Use the uniqueness statement in Theorem 1 in order to get an invariant integral representation affording $\chi$.) Thus we may assume that $G=E$ is perfect. It follows that $G / Z$ is the direct product of the distinct $H$-conjugates of some non-abelian simple group $G_{0} / Z$.

Let $\chi_{0} \in \operatorname{Irr}\left(G_{0}\right)$ denote the irreducible constituent of $\chi_{G_{0}}$. By hypothesis there is an $R$-free $R G_{0}$-lattice $U_{0}$ which affords $\chi_{0}$ and is stable under the inertia group $T_{0}=\mathrm{N}_{H}\left(G_{0}\right)$ of $\chi_{0}$ in $H$. Let $\left\{h_{1}, \cdots, h_{r}\right\}$ be a right transversal to $T_{0}$ in $H$. Then $\chi=\chi_{0}{ }^{h_{1}} \otimes \cdots \otimes \chi_{0}{ }^{h_{r}}$. Consequently $U=U_{0}{ }^{h_{1}} \otimes_{R} \cdots \otimes_{R} U_{0}{ }^{h_{r}}$ is an $R$-free and $H$-stable $R G$-lattice affording $\chi$. We are done.

Fix a group $H$ operating on $G$ and leaving $\chi$ invariant. By Theorem 2 there exists an $H$-stable $R G$-lattice such that $K \otimes_{R} U$ affords $\chi$. The problem is that $U$ need not be $R$-free. We denote by $[U]$ its Steinitz class in the class group $\mathrm{Cl}_{K}$ (cf. Steinitz' theorem in [3, (4.13)]). $U$ is $R$-free if and only if $[U]=1$.

LEMMA. Let $U$ be an $H$-stable $R G$-lattice such that $K \otimes_{R} U$ affords $\chi$.

(a) Every $R G$-lattice $V$ in the genus of $U$ is $H$-stable. In fact, $V \cong J \cdot U$ for some unique (non-zero, fractional) ideal $J$ of $K$, up to $R$-isomorphism, and then $[V]=[J]^{d}[U]$ where $[J]$ is the ideal class of $J$.

(b) If $L / K$ is a finite field extension such that $[U]$ becomes a dth power in $\mathrm{Cl}_{L}$, there exists an $H$-stable representation of $G$ affording $\chi$ over the ring of integers of $L$.

ProOF. Recall that $d=\chi(1)$. Statement (a) is immediate from [3, (31.18) and (31.26)]. Note that $J \cdot U$ is in the genus of $U$ for any ideal $J$ of $K$. Thus (b) follows from (a).

CONJECTURE. Let $p$ be any prime and $k \geq 1$ an integer. Suppose $F$ is an abelian number field containing the $p^{k}$ th but not the $p^{k+1}$ th roots of unity, and let $L=F\left(\varepsilon_{p^{k+1}}\right)$. Then the natural homomorphism $\mathrm{Cl}_{F} \rightarrow \mathrm{Cl}_{L}$ maps every element of $\mathrm{Cl}_{F}$ onto a pth power in $\mathrm{Cl}_{L}$.

This conjecture is confirmed in many instances (see $[8,10,24])$ and is valid in cyclotomic $\mathbb{Z}_{p}$-extensions of abelian number fields at all sufficiently high levels (as 
follows from [11]). It holds true if $F$ occurs in the cyclotomic $\mathbb{Z}_{p}$-extension of a field $K$ (containing the $p$ th roots of unity for odd $p$ and the 4 th otherwise) for which the Iwasawa invariant satisfies $\lambda_{p}(k)<p$.

Recall that $n \cdot d$ is a divisor of $|G|$. If $\chi$ is not of $p$-defect 0 for all primes $p$ dividing $|G|$ and the conjecture holds (for all such $p$ ), we get (stable) realizability of $\chi$ over the ring $\mathbb{Z}\left[\varepsilon_{|G|}\right]$ by the lemma. In case $\chi$ is of $p$-defect 0 for some $p$ dividing $|G|$ additional arguments are necessary to obtain this result. By the proposition it suffices to consider characters of defect 0 of quasisimple groups. Note that most simple groups admit such characters [27].

ProOf OF THEOREM 3'. Let $U$ be as in Theorem 2. Replacing $U$ by a lattice in the same genus, if necessary, we may assume that its Steinitz class $[U]$ has order divisible only by primes dividing $d=\chi(1)$ (see the above lemma).

Let $p$ be any prime dividing $d$. Let $\left\{K_{r}\right\}$ denote the cyclotomic $\mathbb{Z}_{p}$-extension of $K$ and $A_{r}$ the Sylow $p$-subgroup of $\mathrm{Cl}_{K_{r}}$. Since $K$ is an abelian number field, by the theorem of Ferrero-Washington the cyclotomic Iwasawa invariant $\mu_{p}(K)$ equals 0 (cf. [26, Thm. 7.15 and Prop. 13.24]). Thus it follows from the theorem in [11] that for all sufficiently large $r$ the elements in $A_{r}$ become $p$ th powers in $A_{r+1}$. (Indeed the image of $A_{r}$ therein is just the subgroup of all $p$ th powers in $A_{r+1}$.) Hence we find $r=r_{p}$ such that the $p$-part of $[U]$ is a $d$ th power in $A_{r_{p}}$. Denote by $K(p)$ the corresponding field $K_{r_{p}}$.

The compositum $L$ of the $K(p), p$ varying over the primes dividing $d$, has the property that $[U]$ becomes a $d$ th power in its class group. According to the lemma we obtain an $H$-stable representation over the ring of integers of $L$ affording $\chi$. The construction of $L$ (from $K$ ) does not rely on the operator group $H$. Furthermore, $L \subseteq \mathbb{Q}\left(\varepsilon_{n^{\prime}}\right)$ for some integer $n^{\prime}$ divisible only by primes dividing $|G|$. This completes the proof of Theorem $3^{\prime}$.

\section{Some examples}

We are going to substantiate that Theorem $3^{\prime}$ holds for $n^{\prime}=|G|$. By the proposition it suffices to study the situation where $G=G^{\prime}$ is quasisimple and $\chi \in \operatorname{Irr}(G)$ is faithful and $H$-stable, and where $Z=\mathrm{Z}(G)$ is cyclic and central in $H$. Observe that $Z$ is part of the Schur multiplier $\mathrm{M}(\bar{G})$ of the simple group $\bar{G}=G / Z$. Without loss we may assume that $H \supseteq G$ acts faithfully on $\bar{G}$ so that $H / G$ is a subgroup of the group $\operatorname{Out}(\bar{G})$ of outer automorphisms of $\bar{G}$. If $H / G$ is cyclic (or, more generally, has trivial multiplier), $\chi$ can be extended to $H$. By virtue of the classification of the finite simple groups both $\mathrm{M}(\bar{G})$ and $\operatorname{Out}(\bar{G})$ are known. 
Example 1 (Sporadic Groups). Let $\bar{G}$ be a sporadic simple group. Then $\operatorname{Out}(\bar{G})$ has order 1 or 2; in the latter case it inverts the elements in $\mathrm{M}(\bar{G})$ which is cyclic of order $r$, say. There exists then an 'envelope' $r \cdot \bar{G} \cdot 2$ and the isoclinic group $r \cdot \bar{G} \cdot 2 i$ (see the ATLAS [2]). As for class numbers of cyclotomic fields we refer to [26].

In what follows we discuss in detail the cases where the Schur index of the faithful $\chi \in \operatorname{Irr}(G)$ is not trivial (and hence 2), using work of Feit $[6,7]$. Then $\mathbb{Q}(\chi)$ is at most of degree 2 over the rationals and $\Pi(\chi)$, the set of rational places (including $\infty)$ where the local Schur index is non-trivial, consists of at most 2 elements. The remaining cases where the Schur index is trivial are settled by ad hoc considerations (see the table below). The numbering of the characters refers always to the ATLAS [2].

CASE 1. $G=\bar{G}$. Then for all characters under consideration (with non-trivial Schur index) $\mathbb{Q}(\chi)=\mathbb{Q}$, and $\Pi(\chi)=\{2,3\},\{2,5\},\{\infty, 3\}$ or $\{\infty, 5\}$ (Table A in [6]). It follows that $\chi$ can be written in $\mathbb{Q}(\sqrt{3}), \mathbb{Q}(\sqrt{10}), \mathbb{Q}(\sqrt{-3})$ respectively $\mathbb{Q}(\sqrt{-5})$. Here only the field $\mathbb{Q}(\sqrt{-5})$ has nontrivial class number, and in this case $\mathbb{Q}\left(\varepsilon_{20}\right)$ is a splitting field with class number 1 .

If $H=\operatorname{Aut}(G)$ is distinct from $G$ (index 2), both extensions $\chi^{\prime} \in \operatorname{Irr}(H)$ of $\chi$ have Schur index 1. Only the following groups $H$ occur: $J_{2} .2, J_{3} .2$ where $\mathbb{Q}\left(\chi^{\prime}\right)=$ $\mathbb{Q}(\sqrt{6})$, Suz.2 where $\mathbb{Q}\left(\chi^{\prime}\right)=\mathbb{Q}(\sqrt{10})$, and $M^{c} L .2$ [7]. In the last case $\left(G=M^{c} L\right.$ with $\chi=\chi_{11}$ respectively $\chi_{13}$ being of degree 3.520 respectively 4.752 ) we have $\mathbb{Q}(\chi)=\mathbb{Q}$. For $\chi=\chi_{11}$ we have $\Pi(\chi)=\{\infty, 5\}$ and $\mathbb{Q}\left(\chi^{\prime}\right)=\mathbb{Q}(\sqrt{-5})$, otherwise $\Pi(\chi)=\{\infty, 3\}$ and $\mathbb{Q}\left(\chi^{\prime}\right)=\mathbb{Q}(\sqrt{-3})$. Then $\mathbb{Q}\left(\varepsilon_{20}\right)$ respectively $\mathbb{Q}\left(\varepsilon_{24}\right)$ are splitting fields for $\chi^{\prime}$ with class number 1 . In each case there is an representation of $H$ affording $\chi$ ' over some 'small' ring of cyclotomic integers, which gives what we want.

CASE 2. $G=2 . \bar{G}$. Either $\mathbb{Q}(\chi)=\mathbb{Q}$ and $\Pi(\chi)=\{\infty, p\}$ for $p \in\{2,3,5,7,11\}$, or $\Pi(\chi)=\{\infty\}$ and $\mathbb{Q}(\chi)=\mathbb{Q}(\sqrt{k})$ for $k \in\{2,3,5,13,29\}$ (Table $C$ in [6]). In the first case $\chi$ can be written in $\mathbb{Q}\left(\varepsilon_{p}\right)$ for $p \neq 2$ and in $\mathbb{Q}(\sqrt{-2})$ otherwise, which all have class number 1 . In the latter case $\mathbb{Q}\left(\varepsilon_{k}\right)$ for $k \neq 2$, and $\mathbb{Q}\left(\varepsilon_{8}\right)$ otherwise, are splitting fields for $\chi$. Except for $\mathbb{Q}\left(\varepsilon_{29}\right)$ these fields have class number 1 .

In this exceptional case, $G=2 . R u$ and $\chi=\chi_{49}$ resp. $\chi_{50}$, where each is of degree 8.192 with $\mathbb{Q}(\chi)=\mathbb{Q}(\sqrt{29})$. Let then $F(\supset \mathbb{Q}(\chi))$ be the subfield of $\mathbb{Q}\left(\varepsilon_{29}\right)$ which is of degree 4 over the rationals. Since $\Pi(\chi)=\{\infty\}$ and $F$ is not real, $\chi$ can be realized over $F$. Since $\operatorname{Gal}(F / \mathbb{Q})$ is a 2-group and 29 is the unique finite prime which ramifies in $F$, the class number of $F$ is odd [26, Thm. 10.4]. The image of the class group $\mathrm{Cl}_{F}$ in $\mathrm{Cl}_{\mathbb{Q}\left(\varepsilon_{29}\right)}$ is trivial since $\mathbb{Q}\left(\varepsilon_{29}\right)$ has class number 8 .

Let us now investigate invariance. Suppose $H \neq G$ (and $\chi$ is $H$-stable). Then both extensions $\chi^{\prime} \in \operatorname{Irr}(H)$ of $\chi$ have Schur index 2 with $\Pi\left(\chi^{\prime}\right)=\{\infty\}$. With one exception this happens only when $\mathbb{Q}(\chi)=\mathbb{Q}$, with $\mathbb{Q}\left(\chi^{\prime}\right)=\mathbb{Q}(\sqrt{k})$ for $k \in$ 
$\{2,3,5,7,10,11,14,15\}$ (see [7]). The exception is $H=2 . H S .2 i$ where there are the characters $\chi=\chi_{41}$ respectively $\chi_{42}$ of $G=2 . H S$ with degree 2.520 such that $\mathbb{Q}(\chi)=\mathbb{Q}(\sqrt{5})$. Here $\mathbb{Q}\left(\chi^{\prime}\right)=\mathbb{Q}(\sqrt{(5-\sqrt{5}) / 2})$, and for instance $\mathbb{Q}\left(\varepsilon_{40}\right)$ is a splitting field for $\chi^{\prime}$ with class number 1 .

CASE 3. $G=r \cdot \bar{G}$ with $r>2$. Here all faithful $\chi \in \operatorname{Irr}(G)$ have Schur index 1 except for $G=4 . M_{22}$ which admits two Galois conjugate characters $\chi=\chi_{30}$ of degree 176 with $\mathbb{Q}(\chi)=\mathbb{Q}(\sqrt{-1})$ and $\Pi(\chi)=\{5\}$ (Table B in [6]). But then once again $\mathbb{Q}\left(\varepsilon_{20}\right)$ is a splitting field with class number 1 . Since $Z=Z\left(4 . M_{22}\right)$ is not central in $4 . M_{22} .2$ nor in $4 . M_{22} .2 i$, the characters are not stable in these groups. We are done.

In the table below we list only those faithful irreducible characters $\chi$ of $G$ with trivial Schur index where $\chi(1)$ is not prime to the greatest common divisor of the class number $h_{\chi}$ of $\mathbb{Q}(\chi)$ and the class number $h_{f(x)}$ of its conductor (cyclotomic) field. Also, real characters are left out since the class number $h_{f(x)}^{+}$of the maximal real subfield of $\mathbb{Q}\left(\varepsilon_{f(x)}\right)$ is trivial in any instances occurring. The greatest common divisor of $\chi(1), h_{\chi}$ and $h_{f(x)}$ is always a power of some prime $p \in\{2,3,5,7\}$. The $p$-part of $\chi(1)$ is denoted by $\chi(1)_{p}$. The column on the very right (where $n^{\prime}(\chi)$ is listed) describes splitting rings $\mathbb{Z}\left[\varepsilon_{n^{\prime}}\right]$ of cyclotomic integers; $n^{\prime}=n^{\prime}(\chi)$ need not be the best (smallest) possible choice. At any rate, it is always a divisor of $|G|$. Invariance is easily confirmed.

With one exception all fields $\mathbb{Q}(\chi)$ remaining are quadratic or biquadratic. The exception is $G=3 . O^{\prime} N, \chi=\chi_{51}$ where $\mathbb{Q}(\chi)$ is of degree 6 over the rationals. Using the notation of the ATLAS, $\mathbb{Q}\left(c_{19}\right)$ is written for the cubic subfield of $\mathbb{Q}\left(\varepsilon_{19}\right)$, so that $\mathbb{Q}(\chi)=\mathbb{Q}\left(\sqrt{-3}, c_{19}\right)$ here. The cyclotomic Iwasawa invariant $\lambda_{p}=\lambda_{3}$ of this field is 0 whereas $\lambda_{p}(\mathbb{Q}(\chi))=1$ otherwise.

The class numbers (and class groups) of $\mathbb{Q}(\chi)$ are taken from Hasse [13] (and [14]). For the computation of $n^{\prime}(\chi)$ we use various results on cyclotomic $\mathbb{Z}_{p}$-extensions from Washington [26]. In addition, we use arguments from Greenberg [12] and results by Ferrero [8], Gold [10] and Kida [16].

Example 2 (Some Alternating Groups). All irreducible characters of $\bar{G}=A_{k}$ $(k \geq 5)$ have Schur index 1. If $\chi \in \operatorname{Irr}\left(A_{k}\right)$ is invariant under $S_{k}$, it can be realized over $\mathbb{Z}$ by known properties of the symmetric groups. Otherwise $\mathbb{Q}(\chi)$ can be any real quadratic number field. Some characters $\chi$ of $2 . A_{k}$ have Schur index 2 , with $\Pi(\chi)$ being arbitrary large (see Turull [25]).

We only describe briefly the situation for the proper covering groups $G$ of $A_{k}$, $k=5,6,7$, and their faithful characters with non-trivial Schur index (and hence index 2). If $G=2 . A_{5}$ and $\chi \in \operatorname{Irr}(G)$ is its basic spin character, then $\mathbb{Q}(\chi)=\mathbb{Q}(\sqrt{5})$ and 


\begin{tabular}{llllllll}
\hline$G$ & $\chi$ & $\chi(1)_{p}$ & $\mathbb{Q}(\chi)$ & $f(\chi)$ & $h_{\chi}$ & $h_{f(\chi)}$ & $n^{\prime}(\chi)$ \\
\hline & & & & & & & \\
$6 . S u z$ & $\chi_{132}$ & $2^{2}$ & $\mathbb{Q}(\sqrt{-3}, \sqrt{13})$ & 39 & 2 & 2 & $2^{4} \cdot 3 \cdot 13$ \\
$3 . O^{\prime} N$ & $\chi_{51}$ & $3^{4}$ & $\mathbb{Q}\left(\sqrt{-3}, c_{19}\right)$ & 57 & 3 & $3^{2}$ & $3^{2} \cdot 19$ \\
$3 . O^{\prime} N$ & $\chi_{54}$ & $3^{2}$ & $\mathbb{Q}(\sqrt{-3}, \sqrt{-31})$ & 93 & 3 & $3^{2} \cdot 5 \cdot 151$ & $3^{3} \cdot 31$ \\
$3 . F i_{22}$ & $\chi_{117}$ & 2 & $\mathbb{Q}(\sqrt{-3}, \sqrt{13})$ & 39 & 2 & 2 & $2^{3} \cdot 3 \cdot 13$ \\
$6 . F i_{22}$ & $\chi_{192}$ & $2^{5}$ & $\mathbb{Q}(\sqrt{-3}, \sqrt{13})$ & 39 & 2 & 2 & $2^{7} \cdot 3 \cdot 13$ \\
$2 . C o_{1}$ & $\chi_{128}$ & 3 & $\mathbb{Q}(\sqrt{-23})$ & 23 & 3 & 3 & $3^{2} \cdot 23$ \\
$2 . C o_{1}$ & $\chi_{135}$ & $2^{19}$ & $\mathbb{Q}(\sqrt{-14})$ & 56 & $2^{2}$ & 2 & $2^{22} \cdot 7$ \\
$2 . C o_{1}$ & $\chi_{142}$ & $2^{3}$ & $\mathbb{Q}(\sqrt{-39})$ & 39 & $2^{2}$ & 2 & $2^{4} \cdot 3 \cdot 13$ \\
$3 . F_{3+}$ & $\chi_{126}$ & $3^{3}$ & $\mathbb{Q}(\sqrt{-3}, \sqrt{-23})$ & 69 & 3 & $3 \cdot 23$ & $3^{4} \cdot 23$ \\
$3 . F_{3+}$ & $\chi_{138}$ & $3^{15}$ & $\mathbb{Q}(\sqrt{-3}, \sqrt{29})$ & 87 & 3 & $3 \cdot 23$ & $3^{16} \cdot 29$ \\
$B$ & $\chi_{21}$ & $5^{6}$ & $\mathbb{Q}(\sqrt{-47})$ & 47 & 5 & $5 \cdot 139$ & $5^{6} \cdot 11 \cdot 47$ \\
$B$ & $\chi_{25}$ & 3 & $\mathbb{Q}(\sqrt{-23})$ & 23 & 3 & 3 & $3^{2} \cdot 23$ \\
$B$ & $\chi_{45}$ & $3^{13}$ & $\mathbb{Q}(\sqrt{-31})$ & 31 & 3 & $3^{2}$ & $3^{13} \cdot 31$ \\
$B$ & $\chi_{52}$ & 3 & $\mathbb{Q}(\sqrt{-23})$ & 23 & 3 & 3 & $3^{2} \cdot 23$ \\
$2 . B$ & $\chi_{229}$ & $5^{6}$ & $\mathbb{Q}(\sqrt{-47})$ & 47 & 5 & $5 \cdot 139$ & $5^{6} \cdot 11 \cdot 47$ \\
$2 . B$ & $\chi_{238}$ & $3^{13}$ & $\mathbb{Q}(\sqrt{-31})$ & 31 & 3 & $3^{2}$ & $3^{13} \cdot 31$ \\
$M$ & $\chi_{26}$ & $3^{19}$ & $\mathbb{Q}(\sqrt{-31})$ & 31 & 3 & $3^{2}$ & $3^{19} \cdot 31$ \\
$M$ & $\chi_{39}$ & $7^{6}$ & $\mathbb{Q}(\sqrt{-71})$ & 71 & 7 & $7^{2} \cdot 79241$ & $7^{6} \cdot 71$ \\
$M$ & $\chi_{44}$ & 2 & $\mathbb{Q}(\sqrt{-39})$ & 39 & $2^{2}$ & 2 & $2^{3} \cdot 3 \cdot 13$ \\
$M$ & $\chi_{53}$ & $5^{9}$ & $\mathbb{Q}(\sqrt{-47})$ & 47 & 5 & $5 \cdot 138$ & $5^{9} \cdot 11 \cdot 47$ \\
$M$ & $\chi_{71}$ & $3^{18}$ & $\mathbb{Q}(\sqrt{-87})$ & 87 & $2 \cdot 3$ & $2^{9} \cdot 3$ & $3^{19} \cdot 29$ \\
$M$ & $\chi_{74}$ & $5^{9}$ & $\mathbb{Q}(\sqrt{-47})$ & 47 & 5 & $5 \cdot 139$ & $5^{9} \cdot 11 \cdot 47$ \\
$M$ & $\chi_{81}$ & $2^{31}$ & $\mathbb{Q}(\sqrt{-14})$ & 56 & $2^{2}$ & 2 & $2^{34} \cdot 7$ \\
$M$ & $\chi_{83}$ & 3 & $\mathbb{Q}(\sqrt{-23})$ & 23 & 3 & 3 & $3^{2} \cdot 23$ \\
$M$ & $\chi_{89}$ & 2 & $\mathbb{Q}(\sqrt{-95})$ & 95 & $2^{3}$ & $2^{2} \cdot 13 \cdot 19 \cdot 109$ & $2^{3} \cdot 5 \cdot 19$ \\
$M$ & $\chi_{105}$ & $3^{19}$ & $\mathbb{Q}(\sqrt{-31})$ & 31 & 3 & $3^{2}$ & $3^{19} \cdot 31$ \\
$M$ & $\chi_{107}$ & $3^{19}$ & $\mathbb{Q}(\sqrt{-31})$ & 31 & 3 & $3^{2}$ & $3^{19} \cdot 31$ \\
& & & & &
\end{tabular}


$\chi$ can be realized over $\mathbb{Q}\left(\varepsilon_{5}\right)\left[25\right.$, Thm. 5.6]. This $\chi$ is not stable under $2^{ \pm} . S_{5}$. The other characters have similar properties.

All faithful characters of $6 . A_{6}$ and 3. $A_{6}$ have Schur index 1 and their fields of character values have class number 1 (Schur [22]). Some faithful characters of 2. $A_{6}=\mathrm{SL}_{2}(9)$ have Schur index 2 (Feit [6]); they are easily handled. All faithful characters $\chi$ of $6 . A_{7}$ and $3 . A_{7}$ have Schur index 1 and their fields of character values have class number 1 (Schur). Some faithful characters of $2 . A_{7}$ have Schur index 2 (Turull [25]), but they are easily handled.

REMARK. Possibly one can handle the alternating groups and their covering groups by constructing representations in some natural way. A similar statement may be appropriate for the groups of Lie type. We investigated some groups of Lie type of low rank. All the examples computed so far indicate that Theorem 3 ' should hold for $n^{\prime}=|G|$. In order to establish this we would require detailed information on the class groups in the Iwasawa towers at lower levels, in the spirit of the conjecture formulated in Section 5, as well as a better understanding of the integral representation theory of the characters of defect 0 (for the quasisimple groups).

\section{Acknowledgement}

The authors are grateful to J.-P. Serre for pointing out that Iwasawa theory may be helpful, and to Jonathan Sands for the reference to the paper [11] by Grandet and Jaulent.

\section{References}

[1] G. Cliff, J. Ritter and A. Weiss, 'Group representations and integrality', J. Reine Angew. Math. 426 (1992), 193-202.

[2] J. H. Conway, R. T. Curtis, S. P. Norton, R. A. Parker and R. A. Wilson, Atlas of finite simple groups (Clarendon Press, Oxford, 1985).

[3] C. W. Curtis and I. Reiner, Methods of representation theory, I (Wiley, New York, 1981).

[4] E. C. Dade, 'Character values and Clifford extensions for finite groups', Proc. London Math. Soc. 29 (1974), 214-236.

[5] - 'Characters of groups with normal extra special subgroups', Math. Z. 152 (1976), 1-31.

[6] W. Feit, 'The computation of some Schur indices', Israel J. Math. 46 (1983), 274-300.

[7] _ - 'Schur indices of characters of groups related to finite sporadic simple groups', Israel $J$. Math. 93 (1996), 229-251.

[8] B. Ferrero, 'The cyclotomic $\mathbb{Z}_{2}$-extension of imaginary quadratic fields', Amer. J. Math. 102 (1980), 447-459.

[9] M. Schönert et al., GAP - groups, algorithms and programming, version 3, release 4 (Lehrstuhl D für Mathematik, RWTH Aachen, Germany, 1995). 
[10] R. Gold, 'Examples of Iwasawa invariants, II', Acta Arith. 26 (1975), 233-240.

[11] M. Grandet and J.-F. Jaulent, 'Sur la capitulation dans une $\mathbb{Z}_{\ell}$-extension', J. Reine Angew. Math. 362 (1985), 213-217.

[12] R. Greenberg, 'On the Iwasawa invariants of totally real number fields', Amer. J. Math. 98 (1976), 263-284.

[13] H. Hasse, Über die Klassenzahl abelscher Zahlkörper (Akademie-Verlag, Berlin, 1952).

[14] K. Hore and H. Ogura, 'On the ideal class groups of imaginary abelian fields', Trans. Amer. Math. Soc. 347 (1995), 2517-2532.

[15] B. Huppert, Endliche Gruppen, I (Springer, Berlin, 1967).

[16] Y. Kida, 'Cyclotomic $\mathbb{Z}_{2}$-extensions of $J$-fields', J. Number Theory 14 (1982), 340-352.

[17] U. Reinhardt and P. Schmid, 'Invariant lattices and modular decomposition of irreducible representations', J. Algebra 87 (1984), 89-104.

[18] U. Riese and P. Schmid, 'Schur indices and Schur groups, II', J. Algebra 182 (1996), 183-200.

[19] J. Ritter and A. Weiss, 'Galois action on integral representations', J. London Math. Soc. 46 (1992), $411-431$.

[20] P. Schmid, 'The Fong reduction revisited', J. Algebra 162 (1993), 345-354.

[21] — , 'On 2-blocks of characters with defect 1', J. Algebra 183 (1996), 235-244.

[22] I. Schur, 'Über die Darstellungen der symmetrischen und alternierenden Gruppen durch gebrochene lineare Substitutionen', J. Math. 139 (1911), 155-250.

[23] L. Solomon, 'The representations of finite groups in algebraic number fields', J. Math. Soc. Japan 19 (1961), 144-182.

[24] K. Tateyama, 'On the ideal class group of some cyclotomic fields', Proc. Japan Acad. 58, Ser. A (1982), 283-285.

[25] A. Turull, 'The Schur index of projective characters of symmetric and alternating groups', Ann. of Math. (2) 135 (1992), 91-124.

[26] L. C. Washington, Introduction to cyclotomic fields, Graduate Texts in Math. 83 (Springer, Berlin, 1982).

[27] W. Willems. 'Blocks of defect zero in finite simple groups of Lie type', J. Algebra 113 (1988), $511-522$.

Universität Tübingen

Mathematisches Institut

Auf der Morgenstelle 10

D-72076 Tübingen

Germany 